\title{
Les Formes De Relations Client-Fournisseur: Synthese Des Modeles Et Approches Theoriques
}

\author{
Soukaina EL Boujnouni (PhD Student) \\ Department of Management, Faculty of Law, Economics and Social \\ Sciences, Mohammed V University, Agdal, Rabat, Morocco
}

Doi: 10.19044/esj.2018.v14n4p134 URL:http://dx.doi.org/10.19044/esj.2018.v14n4p134

\begin{abstract}
Customer-supplier cooperation is at the heart of industrial concerns, with new market constraints forcing companies to establish stable and lasting relationships with their various partners (Perrotin \& Louberu, 1996). This paper focuses on the requirements of the development of inter-firm exchanges in an industrial environment at a micro level. In particular, we will analyze how client companies structure their relationships with their suppliers and vice versa, and develop mechanisms to control their actions in order to achieve their own goals. We will search through a non-exhaustive list of theoretical approaches to characterize the forms of customer-supplier relationships and the different factors influencing the nature of cooperation between firms. To understand this dynamic, we will then mobilize a theoretical framework of the inter-organizational literature to identify a set of factors favoring the stability and smooth running of an industrial relationship.
\end{abstract}

Keywords: Industry, confidence, control, customer, supplier

\section{Résumé}

Les relations de coopération client-fournisseur sont au cœur des préoccupations industrielles, les nouvelles contraintes du marché forçant les entreprises à établir des relations stables et durables avec leurs différents partenaires (Perrotin \& Louberu, 1996).

Notre travail se focalisera sur les exigences du développement des échanges inter-firmes en milieu industriel à un niveau micro ; en particulier, nous analyserons la manière dont les entreprises clientes structurent leurs relations avec leurs fournisseurs et l'inverse, et développent des mécanismes pour contrôler les actions de ces derniers afin d'atteindre leurs propres buts.

Nous chercherons à travers une liste non exhaustive des approches theoriques à caractériser les formes de relations client-fournisseur et les différents facteurs influant sur la nature de la coopération entre firmes. Pour comprendre 
cette dynamique. Nous mobiliserons ensuite un cadre théorique de la littérature inter-organisationnelle pour identifier un ensemble de facteurs favorisant la stabilité et le bon déroulement d'une relation industrielle.

Mots-clés: Industrie, confiance, contrôle, client, fournisseur

\section{Introduction}

Les relations de coopération client-fournisseur sont au cœur des préoccupations industrielles, les nouvelles contraintes du marché forçant les entreprises à établir des relations stables et durables avec leurs différents partenaires (Perrotin \& Louberu, 1996).

Hamdouch et Depret (2003) affirment que le positionnement concurrentiel des entreprises ne découle plus uniquement de leur capacité à mobiliser des ressources, mais qu'il repose désormais sur leur capacité à gérer des relations d'interdépendance de plus en plus structurelles et étroites avec d'autres partenaires et notamment leurs fournisseurs. Ces relations de coopération clients fournisseurs sont conditionnées par de nombreux facteurs résultant de l'intégration au sein d'un réseau d'acteurs constituant le marché industriel (Salle \& Hugues, 1992).

L'étude du fonctionnement de ces relations inter-organisationnelles nécessite de déterminer les facteurs qui les influencent. Notre travail se focalisera sur les exigences du développement des échanges inter-firmes en milieu industriel à un niveau micro; en particulier, nous analyserons la manière dont les entreprises clientes structurent leurs relations avec leurs fournisseurs et l'inverse, et développent des mécanismes pour contrôler les actions de ces derniers afin d'atteindre leurs propres buts.

Nous chercherons à caractériser les formes de relations clientfournisseur et les différents facteurs influant sur la nature de la coopération entre firmes. Pour comprendre cette dynamique. Nous mobiliserons ensuite un cadre théorique de la littérature inter-organisationnelle pour identifier un ensemble de facteurs favorisant la stabilité et le bon déroulement d'une relation industrielle, nous analyserons ensuite la manière dont les entreprises clientes essaient de structurer leurs relations avec leurs fournisseurs et de développer des mécanismes pour contrôler les actions de ces derniers afin d'atteindre leurs propres buts.

Notre revue de la littérature s'intéresse à l'analyse des relations interorganisationnelles, nous présenterons les différentes représentations conceptuelles de la relation client fournisseur, les facteurs explicatifs de l'existence de ces relations et nous soulignerons les différentes formes qu'elles peuvent prendre. Nous nous intéresserons, ensuite, à la notion de confiance et de contrôle dans les relations client-fournisseur en nous basant sur les différentes théories inter-organisationnelles. 
Sous cet angle, ce travail tente de répondre à la question fondamentale suivante:

Comment, dans un contexte industriel, peut-on comprendre le dynamisme des relations de coopération Clients-fournisseurs?

Cette question principale peut se décliner dans les questions suivantes: - Comment un client sélectionne - il son fournisseur dans l'automobile?

- Quel est le rôle de la confiance dans la structuration de la relation client fournisseur?

- $\quad$ Pourquoi et comment les entreprises clientes contrôlent elles les fournisseurs afin que ceux-ci se comportent conformément à leurs attentes? Avec quels moyens? A quel moment? Quels types de contrôle les clients exercent-ils sur leurs fournisseurs?

L'objectif de ce travail est de présenter un modèle conceptuel préliminaire sur les relations inter organisationnelles, de manière à étudier les relations client-fournisseurs au niveau opérationnel entre les fournisseurs et clients automobiles.

\section{Les Enjeux de la Relation Client-Fournisseur}

Les mutations du marché influencent de manière profonde l'évolution du rapport reliant les donneurs d'ordres avec leurs fournisseurs, et placent les entreprises devant un grand défi de flexibilité et de rapidité pour satisfaire les exigences de leurs partenaires. La sélection d'un fournisseur constitue alors une phase décisive pour la continuité et la stabilité de la relation.

Nogatchewsky (2003, p.173) définit la relation client fournisseur comme " un accord impliquant une relation entre des organisations juridiquement indépendantes qui mettent en commun des actifs (souvent spécifiques) afin d'atteindre des buts communs et individuels.

Selon Fekih-Soussi et Bellon (2004), la sélection des bons partenaires signifie que ces derniers sont prêts à s'engager d'une manière crédible dans la relation, et implique des actions réciproques qui contribuent à garantir la stabilité et la pérennité de la relation.

Oliver (1990) propose six déterminants qui structurent les relations inter-organisationnelles:

La Nécessité Légale: Les organisations développent des relations inter-organisationnelles dans le but de satisfaire à une demande légale d'une autorité supérieure.

L'asymétrie: Les organisations développent des relations avec d'autres organisations pour assurer un contrôle sur les ressources dont elles ont besoin.

La Réciprocité: Les relations inter-organisationnelles reposent davantage sur la coopération afin d'atteindre des buts communs. 
L'efficience: L'organisation cherche à optimiser sa productivité, elle peut recourir à d'autres organisations susceptibles de lui fournir les produits dont elle a besoin à moindre coût.

La Stabilité: Les organisations cherchent à développer des relations inter-organisationnelles stables

La Légitimité: Les organisations développent des relations avec d'autres dans le but d'accroître leur réputation, leur image.

Ces six raisons montrent pourquoi une organisation choisie d'avoir des relations durables et formalisées avec d'autres entreprises. Ce choix peut viser à satisfaire certains besoins, notamment au niveau des ressources, former un certain contrôle sur ses relations d'affaire, afin d'éviter la dépendance, élaborer différentes formes de collaboration, rechercher une certaine stabilité.

\section{Les Formes de Relations Clients Fournisseurs}

Les relations entre clients et fournisseurs peuvent prendre plusieurs formes selon le type et la nature de la relation choisie. Capet et Hoflack (1978) spécifient trois formes de relations en sous-traitance :

"Relation de Confiance: Les contacts sont très étroits au niveau de la planification générale, des ateliers, de la conception et pas seulement au niveau des achats et de la vente;

Relation de "Tutoring»: Le donneur d'ordres aide le sous-traitant en contrôlant la qualité et l'avancement des travaux;

Relation de Représailles: On applique le système de sanction et on abandonne le sous-traitant de manière temporaire ou définitive.

Il est possible, dans certains cas, qu'un client intègre en même temps les trois formes de relations de sous-traitance pour les mêmes produits ou services.

Après avoir présenté les raisons qui peuvent expliquer la formation des relations client-fournisseur. Nous proposons d'approfondir ensuite le rôle de la confiance dans les relations clients fournisseurs et les mécanismes de contrôle dans les relations inter-organisationnelles.

\section{La Confiance dans Les Relations Inter-Organisationnelles}

La confiance est considérée comme un élément fondamental à l'échange économique (Zucker, 1986) et même le fondement de l'ordre social (Granovetter, 1985) et des relations interpersonnelles.

Développé par des sociologues dans le cadre de relations interpersonnelles, ce concept a été diffusé aux disciplines liéés aux sciences humaines pour devenir aujourd'hui une dimension importante des problématiques inter-organisationnelles (Sako, 1999). Est considéré comme l'un des facteurs les plus susceptibles d'influencer le déroulement d'une relation client fournisseur (Sako, 1992; Williamson, 1993). 
L'objectif de cette partie est d'étudier le rôle de la confiance dans les relations client-fournisseur et plus particulièrement son influence sur l'engagement que développent les clients à l'égard de leurs fournisseurs ou l'inverse.

Dans un premier temps, le concept de confiance sera défini afin d'identifier ses multiples dimensions. Nous présenterons par la suite les différentes formes de confiance, ensuite analyser son influence sur l'engagement que développent les clients à l'égard de leurs fournisseurs ou l'inverse:

- Quel est le rôle de la confiance dans la structuration de la relation client-fournisseur et dans le choix de tel ou tel fournisseur?

- Comment le degré de confiance se traduit-il concrètement?

\section{Définitions et Attributs de la Confiance}

Les définitions de la confiance sont multiples vu la diversité de courants théoriques et de disciplines intéressés à ce concept. Il s'agit d'une variable multidimensionnelle (Lewis \& Weigert, 1985) qui se définit en référence au contexte dans lequel elle est appliquée.

Définie comme une attente (Frisou, 2000), une intention de comportement ou un sentiment, la confiance est un construit polysémique dont la définition dépend du contexte dans lequel elle est étudiée (Guibert, 1999).

Ring et Van de Ven (1992), considèrent la confiance comme " un mode de coordination spécifique, alternatif au contrat ». Un niveau élevé de confiance, permet aux parties de se concentrer sur les bénéfices à long terme de la relation.

Deutsh (1958) définit la confiance en fonction des motivations des parties. Un agent n'accorde sa confiance que s'il pense que des conséquences avantageuses pour lui pourraient en découler, elle permet de minimiser les risques de comportements opportunistes de la part du partenaire, tout en diminuant les coûts liés à la transaction (Neuville, 1998).

Ces diverses définitions de la confiance montrent que c'est une notion multidimensionnelle nécessitant divers niveaux d'analyse, elle est basée sur les attentes et les comportements des acteurs qui se manifestent dans les relations interentreprises, c'est une forme d'interaction dans laquelle les acteurs s'engagent moralement et de bonne volonté.

\section{Caractéristiques}

Les organisations actuelles sont encastrées dans un contexte social et institutionnel donné, Granovetter $(1985,1992)$ s'intéresse au rôle des relations personnelles et aux structures de ces relations pour générer la confiance, autrement dit la préférence de travailler avec un partenaire avec des relations développées pour des transactions satisfaisantes sur le volet social et 
économique, donc il est primordial d'élargir la dimension relationnelle aux parties prenantes.

La confiance est essentielle dans la phase d'exploration entre les acteurs car elle permet à une relation de passer du stade de la simple connaissance à celui de l'engagement, elle est présente à chaque stade du développement d'une relation.

Après une revue des définitions du concept, nous étudierons les niveaux de la confiance, ses types, puis ses implications pour les relations inter-organisationnelles.

Barney et Hansen (1994) distinguent entre trois niveaux de confiance: " - La confiance faible, caractéristique des transactions dans des marchés très compétitifs, où les occasions d'opportunisme seront limitées ;

- La confiance semi-forte, qui repose sur des mécanismes de gouvernance identifiant les comportements opportunistes potentiels;

- La confiance forte, il s'agit d'une confiance de principe. Elle trouve ses origines dans les principes et valeurs que les partenaires amènent dans l'échange. »

La confiance représente un facteur indispensable dans le développement de la relation client fournisseur, il convient de la créer ou de la renforcer. L'un des moyens de faire consiste à créer des liens étroits via l'engagement crédible qui renvoie à «l'intention pour un membre de continuer la relation » (Anderson, E. \& Weitz, 1989, p.67). L'engagement apparait ainsi comme un élément fondamental à la durabilité d'un partenariat, et se traduit par une intention de maintenir la relation sur le long terme.

Sako (1992), pour sa part, identifie trois types de confiance:

" - La confiance contractuelle, qui a comme base les standards moraux d'honnêteté donnés dans le contrat;

- La confiance de compétences, repose sur l'assurance que le partenaire a les prérequis nécessaires au bon déploiement du partenariat;

- La confiance de bienveillance c'est-à-dire la perception que le partenaire agira dans l'intérêt de la relation et non de façons individuelle ».

On peut conclure que la confiance est un dispositif de coordination fondamental à toutes formes de structuration sociale, particulièrement les partenariats où elle est importante puisqu'elle vise à diminuer l'incertitude.

\section{Contrôle dans Les Relations Inter-Organisationnelles}

Au cours des vingt dernières années, les entreprises ont augmenté l'externalisation de leurs activités à travers la collaboration avec d'autres organisations (Donada \& Nogatchewsky, 2005; Dyer, 1997) par exemple un fournisseur peut décider d'externaliser une partie de sa production c'est notamment le cas dans l'industrie automobile. 
Les entreprises organisent les échanges avec leurs fournisseurs en développant des modes de coopération, mais aussi des processus de contrôle inter-organisationnels. En effet les fournisseurs représentent une catégorie hétérogène formée de firmes qui se différencient par leur taille, leurs compétences technologiques et leur environnement concurrentiel (Doleschal, 1989; Enrietti, 1989). Il s'agit de l'approche relationnelle qui met en exergue l'importance de la collaboration entre organisations au cours d'un échange et la nécessité de développer la confiance entre chaque partenaires pour maximiser les bénéfices résultant de la collaboration,

- Comment s'organise le contrôle au sein d'une relation client fournisseur?

- Pourquoi et comment les clients contrôlent-ils leurs fournisseurs?

- Quels types de contrôle les clients exercent-ils sur leurs fournisseurs?

- Quelles sont les alternatives possibles en termes d'outils et de mécanismes de contrôle pour contribuer plus efficacement à la réussite des relations inter-organisationnelles?

Nous procédons à une revue de la littérature sur la notion de contrôle et les mécanismes susceptibles de gérer les relations interorganisationnel.

\section{Définitions}

Le contrôle dans le cadre d'une relation client-fournisseur est défini comme "le processus par lequel le client influence les actions de son fournisseur dans le sens de ses attentes. " (Donada \& Nogatchewsky, 2005, p.64).

Das et Teng (1998) définissent également le contrôle comme un processus de standardisation par lequel les éléments d'un système deviennent plus prévisibles suite l'établissement de standards. Quand une partie exerce un contrôle sur une autre partie, influence ou définit ses actions afin de réaliser les résultats estimés elle exerce son pouvoir (Frazier, 1983).

Le contrôle est considéré comme le processus par lequel le client oriente et influence les actions du fournisseur afin de satisfaire ses attentes.

\section{Fondements Théoriques D'un Contrôle Inter-Organisationnel}

Cette partie présente les courants principaux étudiant la question des modes de contrôle inter-organisationnels: la théorie de l'agence, la théorie des coûts de transaction.

Nous soulignons les différents facteurs influant sur la nature de la coopération dans relations inter-organisationnelles entre un client et un fournisseur automobile. 


\section{La Théorie de L'agence}

La théorie de l'agence apporte un cadre conceptuel appliqué en contrôle organisationnel et en sociologie pour expliquer les relations entre propriétaires et dirigeants, elle se fonde sur la légitimité du droit à la propriété.

La relation d'agence est un " contrat dans lequel une personne (ou plusieurs), appelée principal, a recours aux services d'une autre personne, l'agent, pour accomplir en son nom une tâche quelconque, ce qui implique une délégation de nature décisionnelle à l'agent » (Jensen \& Meckling, 1976, p. 308).

Le recours à la théorie de l'agence permet de considérer que la délégation à un acteur externe provoque une relation d'agence singulière, les agents signent des contrats qui limitent leurs comportements et mettent en place des dispositifs de coordination basés sur des obligations mutuels.

Une relation de coopération donne lieu à un problème d'agence, la divergence d'intérêts entre les deux acteurs et l'incertitude associée au comportement de l'agent. En effet, si la coopération se déroule dans un cadre certain où tous les comportements sont observables, un contrat classique ressoudra le problème de la divergence d'intérêts.

La théorie de l'agence permet d'analyser aussi bien les relations interorganisationnelles qu'intra-organisationnelles. Elle s'applique aux relations inter-organisationnelles car chaque entité peut poursuivre des objectifs différents, et de ce fait être en conflit sur les raisons de la coopération. Elle permet aussi d'analyser les relations entre individus au sein de l'organisation, dont les intérêts peuvent différer de ceux du dirigeant.

\section{La Théorie de Coûts de Transaction}

Toute transaction génère un coût qui vient s'ajouter au seul coût du produit échangé, la théorie des coûts de transaction a en partie pour origine les travaux de Coase (1937) utilisant la transaction comme point de référence.

Williamson poursuivra la voie ouverte par Coase (1937), en proposant une théorie des coûts de transaction. L'auteur s'intéresse aux facteurs permettant aux individus d'organiser leurs relations contractuelles et plus largement à ceux encadrant leurs transactions (les institutions de l'économie). Il en déduira un ensemble d'applications sur les formes d'organisation, sur l'intégration verticale et sur les frontières de la firme. Pour WILLIAMSON, l'entreprise est une cellule autonome dont la coordination des activités économiques s'exerce par des relations d'autorité. Le marché est lui aussi un lieu de coordination mais fondé sur l'échange et le contrat. Les coûts de transaction sont définis comme «les coûts de fonctionnement du système économique qui doivent être distingués des coûts de production, catégorie de coût dont s'est préoccupée l'analyse néo-classique »(Williamson, 1994). 
Il développera dans les années 1970 et 1980 une analyse des mécanismes d'allocation «hiérarchique» des ressources, il propose différents arrangements institutionnels pour gérer les transactions de manière efficiente.

La théorie des coûts de transaction est une approche institutionnelle de l'organisation économique qui s'attache à déterminer la structure de gouvernance optimale permettant de réduire les couts de transaction pour une tache donnée. (Williamson, 1996, p. 136).

L'objectif de cette approche est de proposer un mode de gouvernance qui permet de minimiser le coût des transactions. Trois modes de gouvernance sont proposés : le marché, la forme hybride et la hiérarchie.

Dans la gouvernance par le marché, le contrôle provient de la concurrence libre et parfaite, le type de contrat dans cette forme est classique. La rupture d'une relation n'a pas de conséquences.

puisqu'une nouvelle relation peut commencer à tout moment dans les mêmes conditions. Dans la gouvernance hybride, le contrôle provient du contrat néo-classique dans lequel chaque partie accepte l'assistance d'un tiers (un arbitre) dans la résolution des conflits et l'évaluation de l'exécution. D'autres mécanismes de sauvegarde permettent de renforcer sa nonremplaçabilité ou, tout au moins, d'augmenter les coûts de changement de partenaire. Dans la gouvernance hiérarchique, le contrôle provient de dispositifs contractuels personnalisés pour lesquels le système de sanction/récompense est à long terme ainsi que de mécanismes hiérarchiques tels que l'autorité.

Dans le cadre de relations interentreprises, ces contrats sont des moyens contre l'opportunisme des agents dans des situations d'incertitude et de divergence d'intérêts.

Nous avons choisi de nous intéresser à deux courants de recherche principaux étudiant la question des modes de contrôle interorganisationnels : la théorie de l'agence, la théorie des coûts de transaction .Les deux cherchent à expliquer le fonctionnement des organisations en partant de la personne physique. L'individu, supposé rationnel, cherche à atteindre un objectif, sur la base d'un calcul ; les actions de la firme correspondent à ce que celui-ci considère comme efficace pour atteindre ces objectifs. Ces deux approches nous fournissent un éclairage essentiel pour étudier le contrôle dans la relation client-fournisseur en termes de choix de modes de gouvernance et de mécanismes de contrôle optimaux.

\section{Les Mécanismes de Contrôle}

Williamson (1981a, 1985) identifie trois grands types de mécanismes de contrôle: 
"- Le contrôle bureaucratique, reposant sur un pilotage direct ou mécanique et le développement de routines et procédures en amont; exemple : les audits...

- Le contrôle marchand, reprenant la notion de régulation par la loi de l'offre et de la demande, ainsi que l'utilisation de procédures d'incitations et de sanctions ;

- Et le contrôle social, de nature plus informelle, reposant sur les relations interpersonnelles entre acteurs. "

Ouchi $(1977,1980)$ pour sa part distingue trois modes de contrôle (par le marché, la bureaucratie ou la culture) généralement exercés par les partenaires aux relations interorganisationnelles.

Le contrôle par le marché se base sur la libre concurrence. Il implique une transaction entre deux entités régulée par un mécanisme de prix comme une source d'information dont dispose le client pour sélectionner son fournisseur., aucun mécanisme de contrôle spécifique n'est nécessaire puisque le client a la possibilité de changer de fournisseur à tout moment.

Le contrôle bureaucratique se base sur ce sont des mécanismes de contrôle formels et hiérarchiques, basés sur les règles, la surveillance, l'évaluation, la direction a et le pouvoir formel .Ce mode de contrôle se révèle par un accompagnement quotidien du client qui peut s'étendre à toute l'organisation du fournisseur, soit un contrôle par l' " input » (les mécanismes bureaucratiques, par la procédure ou sur le comportement direct) soit un contrôle par l' " output » (le contrôle par les résultats permettant de comparer les résultats atteints par rapport à ceux attendus).

Le contrôle par la confiance ou contrôle social et culturel repose sur des mécanismes de surveillances informelles comme la confiance la coopération et les bonnes relations comportementales. Ouchi (1979) stipule que le contrôle social est adapté aux situations où il y a confiance et permet de la même de nourrir la confiance. Les fournisseurs sont retenus sur la base de leur réputation, et sur la base des expériences passées dans lesquelles ils se sont révélés dignes de confiance. Ce type de contrôle nécessite le temps en raison de la difficulté à développer rapidement une relation.

Dans la lignée des travaux d'Ouchi $(1977 ; 1979)$ les acteurs poursuivent leurs intérêts propres

Comme nous pouvons le voir, cette typologie se référant à la coentreprise est applicable à la relation client-fournisseur. En effet, il s'agira d'identifier les éléments principaux, mais aussi l'étendue du contrôle que voudra exercer le client afin d'identifier les mécanismes correspondant à chaque configuration. 


\section{Conclusion}

Cet article était consacré aux formes de relations client-fournisseur. Tout d'abord, nous avons explicité les raisons pour lesquelles une entreprise peut être amenée à entrer en relation avec d'autres, ce qui limite par ailleurs ses libertés d'action et la rend plus vulnérable. Nous avons ensuite recensé les concepts et les modèles permettant de théoriser la relation de, une autre section était dédiée à l'étude des mécanismes de contrôle dans les relations client fournisseur. Nous avons présenté les propositions de la théorie de l'agence et la théorie des couts de transactions appliquées aux relations client-fournisseur.

\section{References:}

1. Barney, J.B. \& Hansen, M.H. (1994). "Trustworthiness as a source of competitive advantage", Strategic Management Journal, 15, p. 175190.

2. Birnbirg, J. (1998). 'Control in Interfirm Co-Operative Relationships', Journal of Management Studies, 35 (4): 421-428.

3. Capet Marcel \& Hoflack Jean (1978). Gestion de l'entreprise de soustraitance, Economica, 1978, Paris, 172 pages.

4. Granovetter, M. (1985). "Economic action and social structure the problem of embeddedness", American Journal of Sociology, vol. 91, $\mathrm{n}^{\circ} 3,1985$, p. 481-510.

5. Gwena“elle Nogatchewsky (2004). Les configurations de contr^ole dans les relations client-fournisseur. Gestion et management. Universit'e Paris Dauphine - Paris IX, Fran,cais.

6. Hamdouch, A. \& Depret, M.-H. (2001). La nouvelle économie industrielle de la pharmacie, Elsevier, Paris.

7. Kœnig, G. (1994). «Introduction : repérage des lieux », Dossier «L'apprentissage organisationnel: pratiques et théories », Revue française de gestion, $\mathrm{n}^{\circ}$ 97, janvier-février, p. 78 .

8. Oliver, C. (1990). Determinants of interorganizational relationships: integration and future directions. Academy of Management Review, v. 15 , n. 2, p. 241-265.

9. Ouchi, W.G. (1980). Markets, Bureaucracies and Clan, Administrative Science Quarterly, 25, 1, 129-141.

10. Perrotin, R. \& Louberu, J-M. (1996). Nouvelles strategies d'achat: sous-traitance, cooperation, partenariat?, Paris, Editions d'Organisation

11. Ring, G.P. \& Van de Ven A.H. (1992). "Structuring cooperative relationship between organization", Strategic Management Journal, vol. 13 , p. 483-498.

12. Sako, M. (1999). Does Trust Improve Business Performance”, in Trust Within and Between Organizations, Oxford University Press. 
13. Sako, M.P. (1992). "Quality and Trust. Interfirm Relations in Britain and Japan", Cambridge University Press, Cambridge.

14. Williamson, O. (1993). "Calculativeness, Trust and Economic Organization", Journal of Law and Economics, XXXVI, pp 453-486.

15. Williamson, O.E. (1975). Markets and Hierarchies: Analysis and Antitrust Implications, New York, The Free Press.

16. Zucker, L. (1986). "Production of trust: institutional sources of economic structure :1840- 1920", Research in Organization Behaviour, 8, pp 53-111. 